\title{
Hereditarily Hurewicz spaces and Arhangel'skiĭ sheaf amalgamations
}

Received January 9, 2008 and in revised form December 31, 2009

\begin{abstract}
A classical theorem of Hurewicz characterizes spaces with the Hurewicz covering property as those having bounded continuous images in the Baire space. We give a similar characterization for spaces $X$ which have the Hurewicz property hereditarily.

We proceed to consider the class of Arhangel'skiı $\alpha_{1}$ spaces, for which every sheaf at a point can be amalgamated in a natural way. Let $C_{p}(X)$ denote the space of continuous real-valued functions on $X$ with the topology of pointwise convergence. Our main result is that $C_{p}(X)$ is an $\alpha_{1}$ space if, and only if, each Borel image of $X$ in the Baire space is bounded. Using this characterization, we solve a variety of problems posed in the literature concerning spaces of continuous functions.
\end{abstract}

Keywords. Pointwise convergence, point-cofinite covers, $\alpha_{1}$, eventual dominance, Hurewicz property, selection principles, QN sets, ideal convergence

\section{Contents}

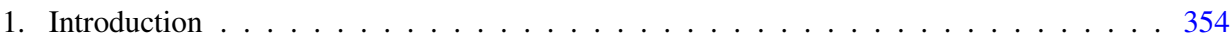

2. A characterization of hereditarily Hurewicz spaces . . . . . . . . . . . . . 355

3. Bounded Borel images . . . . . . . . . . . . . . . . . . . . . . . . . 358

4. Applications . . . . . . . . . . . . . . . . . . . . . . . 362

4.1. QN spaces . . . . . . . . . . . . . . . . . . . . . . . 362

4.2. Convergent sequences of Borel functions . . . . . . . . . . . . . . . . . . . . . 364

4.3. Almost continuous functions . . . . . . . . . . . . . . . . . . . 364

4.4. wQN spaces and the Scheepers Conjecture . . . . . . . . . . . . . . . . . . . . 364

4.5. $\overline{\mathrm{QN}}$ spaces and $M$ spaces . . . . . . . . . . . . . . . . . . . . . . . . 365

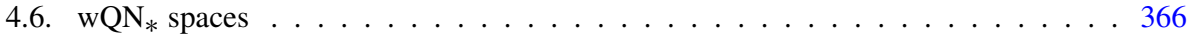

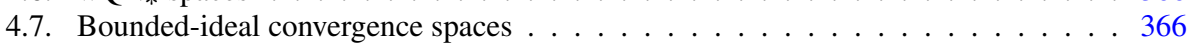

4.8. Bounded Baire-class $\alpha$ images . . . . . . . . . . . . . . . . . . . 368

5. Closing the circle: Continuous bounded images again . . . . . . . . . . . . . . . . 369

References . . . . . . . . . . . . . . . . . . . . . 370

B. Tsaban: Department of Mathematics, Bar-Ilan University, Ramat-Gan 52900, Israel, and Department of Mathematics, Weizmann Institute of Science, Rehovot 76100, Israel; e-mail: tsaban@math.biu.ac.il

L. Zdomskyy: Department of Mathematics, Weizmann Institute of Science, Rehovot 76100, Israel; current address: Kurt Gödel Research Center for Mathematical Logic, Universität Wien, Währinger Str. 25, A-1090 Wien, Austria; e-mail: lzdomsky@gmail.com

Mathematics Subject Classification (2010): Primary 37F20; Secondary 26A03, 03E75 


\section{Introduction}

We are mainly concerned with spaces $X$ which are (homeomorphic to) sets of irrational numbers, and we recommend adopting this restriction for clarity. Our results (and proofs) apply to all topological spaces $X$ in which each open set is a union of countably many clopen sets, and the spaces considered are assumed to have this property. ${ }^{1}$

Fix a topological space $X$. Let $\mathscr{A}, \mathscr{B}$ be families of covers of $X$. The space $X$ may or may not have the following property [35].

$\mathrm{U}_{\text {fin }}(\mathscr{A}, \mathscr{B}):$ Whenever $\mathcal{U}_{1}, \mathcal{U}_{2}, \ldots \in \mathscr{A}$ and none contains a finite subcover, there exist finite sets $\mathcal{F}_{n} \subseteq \mathcal{U}_{n}, n \in \mathbb{N}$, such that $\left\{\bigcup \mathcal{F}_{n}: n \in \mathbb{N}\right\} \in \mathscr{B}$.

Let $\mathrm{O}$ denote the collection of all countable open covers of $X{ }^{2}$ A cover $\mathcal{U}$ of $X$ is pointcofinite if $\mathcal{U}$ is infinite and each $x \in X$ is a member of all but finitely many members of $\mathcal{U}$. ${ }^{3}$ Let $\Gamma$ denote the collection of all open point-cofinite covers of $X$. Motivated by studies of Menger [26], Hurewicz [19] introduced the Hurewicz property $\mathrm{U}_{\text {fin }}(\mathrm{O}, \Gamma)$.

Hurewicz [19] essentially obtained the following combinatorial characterization of $\mathrm{U}_{\text {fin }}(\mathrm{O}, \Gamma)$ (see Recław [29]). For $f, g \in \mathbb{N}^{\mathbb{N}}, f \leq^{*} g$ means $f(n) \leq g(n)$ for all but finitely many $n$. A subset $Y$ of $\mathbb{N}^{\mathbb{N}}$ is bounded if there is $g \in \mathbb{N}^{\mathbb{N}}$ such that $f \leq{ }^{*} g$ for all $f \in Y$.

Theorem 1 (Hurewicz). X satisfies $\mathrm{U}_{\text {fin }}(\mathrm{O}, \Gamma)$ if, and only if, every continuous image of $X$ in $\mathbb{N}^{\mathbb{N}}$ is bounded.

This characterization has found numerous applications—see [38, 24, 41] and references therein. We give a similar characterization for hereditarily Hurewicz spaces, that is, spaces $X$ such that each subspace of $X$ satisfies $U_{\text {fin }}(O, \Gamma)$.

The property of being hereditarily Hurewicz was studied in, e.g., [15, 28, 27]. Rubin introduced a property of subsets of $\mathbb{R}$ such that the existence of a set with this property is equivalent to the possibility of a certain construction of boolean algebras [31]. Miller [27] proved that the Rubin spaces are exactly the hereditarily Hurewicz spaces.

The property of being hereditarily Hurewicz also manifests itself as follows: A set $X \subseteq \mathbb{R}$ is a $\sigma^{\prime}$ space [32] if for each $F_{\sigma}$ set $E$, there is an $F_{\sigma}$ set $F$ such that $E \cap F=\emptyset$ and $X \subseteq E \cup F$. This property was effectively used in studies of generalized metric spaces [13]. Recently, Sakai proved that $X$ is a $\sigma^{\prime}$ space if, and only if, $X$ is hereditarily Hurewicz (Theorem 6 below).

There exist additional classes of hereditarily Hurewicz spaces in the literature. We describe some of them.

A topological space is Fréchet if each point in the closure of a subset of the space is a limit of a convergent sequence of points from that subset. The following concepts,

\footnotetext{
1 Every perfectly normal space (open sets are $F_{\sigma}$ ) with upper inductive dimension 0 (disjoint closed sets can be separated by a clopen set) has the required property. Thus, the spaces considered in the references also have the required property.

2 If $X$ is Lindelöf, we can consider arbitrary open covers of $X$.

3 Traditionally, point-cofinite covers were called $\gamma$-covers [17].
} 
due to Arhangel'skiı̌ [1, 2], are important in determining when a product of Fréchet spaces is Fréchet. Let $Y$ be a general topological space (not necessarily Lindelöf or zerodimensional). A sheaf at a point $y \in Y$ is a family of sequences, each converging to $y$. To avoid trivialities, we consider only sequences of distinct elements. We say that a countable set $A$ converges to $y$ if some (equivalently, each) bijective enumeration of $A$ converges to $y$. The space $Y$ is an $\alpha_{1}$ space if for each $y \in Y$, each countable sheaf $\left\{A_{n}: n \in \mathbb{N}\right\}$ at $y$ can be amalgamated as follows: There are cofinite subsets $B_{n} \subseteq A_{n}, n \in \mathbb{N}$, such that the set $B=\bigcup_{n} B_{n}$ converges to $y$. The references dealing with $\alpha_{1}$ spaces are too numerous to be listed here; see [40] and the references therein for a partial list.

Fix a space $X$. Denote by $C_{p}(X)$ the family of all continuous real-valued functions on $X$, viewed as a subspace of the Tychonoff product $\mathbb{R}^{X}$. A sequence of results by Bukovský-Recław-Repický [10], Recław [30], Sakai [33], and Bukovský-Haleš [9], culminated in the result that if $C_{p}(X)$ is an $\alpha_{1}$ space, then $X$ is hereditarily Hurewicz. Our main result is that if $C_{p}(X)$ is an $\alpha_{1}$ space, then each Borel image of $X$ in $\mathbb{N}^{\mathbb{N}}$ is bounded. It is easy to see that the converse implication also holds, and we obtain a powerful characterization of spaces $X$ such that $C_{p}(X)$ is an $\alpha_{1}$ space.

Historically, the realization that if $C_{p}(X)$ is an $\alpha_{1}$ space then $X$ is hereditarily $\mathrm{Hu}$ rewicz goes through QN spaces [10]: Let $Y$ be a metric space. A function $f: X \rightarrow Y$ is a quasi-normal limit of functions $f_{n}: X \rightarrow Y$ if there are positive reals $\epsilon_{n}, n \in \mathbb{N}$, converging to 0 such that for each $x \in X, d\left(f_{n}(x), f(x)\right)<\epsilon_{n}$ for all but finitely many $n$. A topological space $X$ is a $Q N$ space if whenever 0 is a pointwise limit of a sequence of continuous real-valued functions on $X$, then 0 is a quasi-normal limit of that sequence. QN spaces are studied in, e.g., [10, 30, 36, 28, 11, 33, 9]. In [33, 9] it was shown that $X$ is a QN space if, and only if, $C_{p}(X)$ is an $\alpha_{1}$ space. Thus, QN spaces are also characterized by having bounded Borel images in $\mathbb{N}^{\mathbb{N}}$.

We use our main theorem to show that quite a few additional properties studied in the literature are equivalent to having bounded Borel images in $\mathbb{N}^{\mathbb{N}}$, and consequently solve a variety of problems posed in the literature. To make the paper self-contained and accessible to a wide audience, we supply proofs for all needed results. Often, our proofs of known results are slightly simpler than those available in the literature.

\section{A characterization of hereditarily Hurewicz spaces}

Let $\overline{\mathbb{N}}=\mathbb{N} \cup\{\infty\}$ be the one-point compactification of $\mathbb{N}$, and endow $\overline{\mathbb{N}}^{\mathbb{N}}$ with the Tychonoff product topology. An element $f \in \overline{\mathbb{N}}^{\mathbb{N}}$ is eventually finite if there is $m$ such that $f(n)<\infty$ for each $n \geq m$. Let $\mathbb{E F}$ be the subspace of $\overline{\mathbb{N}}^{\mathbb{N}}$ consisting of all eventually finite elements of $\overline{\mathbb{N}}^{\mathbb{N}}$. The relation $\leq$ * extends to $\mathbb{E} \mathbb{F}$ in the natural way.

Theorem 2. $X$ is hereditarily $\mathrm{U}_{\mathrm{fin}}(\mathrm{O}, \Gamma)$ if, and only if, every continuous image of $X$ in $\mathbb{E} \mathbb{F}$ is bounded.

Proof. $(\Rightarrow)$ Assume that $\Psi: X \rightarrow \mathbb{E} \mathbb{F}$ is continuous. For each $n$, define the following $\left(G_{\delta}\right)$ subset of $\Psi[X]$ :

$$
G_{n}=\{f \in \Psi[X]:(\forall m \geq n) f(m)<\infty\} .
$$


Let $T^{n}: G_{n} \rightarrow \mathbb{N}^{\mathbb{N}}$ be the shift transformation defined by $T^{n}(f)(m)=f(m+n)$ for all $m$.

For each $n, X_{n}=\Psi^{-1}\left[G_{n}\right] \subseteq X$, and therefore $X_{n}$ satisfies $\mathrm{U}_{\text {fin }}(\mathrm{O}, \Gamma)$. By Theorem $1, T^{n}\left[\Psi\left[X_{n}\right]\right]=T^{n}\left[G_{n}\right]$ is a bounded subset of $\mathbb{N}^{\mathbb{N}}$. Thus, $G_{n}$ is a bounded subset of $\mathbb{E} \mathbb{F}$, and therefore so is $\Psi[X]=\bigcup_{n} G_{n}$.

$(\Leftarrow)$ First, note that Hurewicz's Theorem 1 and our assumption on $X$ imply that $X$ satisfies $\mathrm{U}_{\text {fin }}(\mathrm{O}, \Gamma)$.

Lemma 3. If each $G_{\delta}$ subset of $X$ satisfies $\mathrm{U}_{\mathrm{fin}}(\mathrm{O}, \Gamma)$, then $X$ is hereditarily $\mathrm{U}_{\mathrm{fin}}(\mathrm{O}, \Gamma)$.

Proof. Let $Y \subseteq X$. Assume that $\mathcal{U}_{n}, n \in \mathbb{N}$, are covers of $Y$ by open subsets of $X$, which do not contain finite subcovers. Each $\mathcal{U}_{n}$ is an open cover of $G=\bigcap_{n} \cup \mathcal{U}_{n} \supseteq Y$, and has no finite subcover of $G$. As $G$ is a $G_{\delta}$ subset of $X$, it satisfies $\mathrm{U}_{\text {fin }}(\mathrm{O}, \Gamma)$. Thus, there are finite $\mathcal{F}_{n} \subseteq \mathcal{U}_{n}, n \in \mathbb{N}$, such that $\left\{\bigcup \mathcal{F}_{n}: n \in \mathbb{N}\right\}$ is a point-cofinite cover of $G$, and therefore of $Y$.

Assume that $G$ is a $G_{\delta}$ subset of $X$.

Lemma 4 (Sakai [33]). For each $G_{\delta}$ subset $G$ of $X$, there is an open point-cofinite cover $\left\{U_{n}: n \in \mathbb{N}\right\}$ of $X$ such that $G=\bigcap_{n} U_{n}$.

Proof. We have $G^{\mathrm{c}}=\bigcup_{n} C_{n}$ with each $C_{n}$ closed. If $A$ is closed and $B$ is open, then $B$ is a union of countably many disjoint clopen sets, and therefore $A \cap B$ is a union of countably many disjoint closed sets. Thus, each of the disjoint sets $C_{n} \backslash\left(C_{1} \cup \cdots \cup C_{n-1}\right)$, $n \in \mathbb{N}$, is a union of countably many disjoint closed sets. Hence, $G^{\mathrm{c}}=\bigcup_{n} \tilde{C}_{n}$ where the sets $\tilde{C}_{n}$ are closed and disjoint, and therefore $G=\bigcap_{n} \tilde{C}_{n}^{c}$, where $\left\{\tilde{C}_{n}^{c}: n \in \mathbb{N}\right\}$ is an open point-cofinite cover of $X$.

So, let $\left\{U_{n}: n \in \mathbb{N}\right\}$ be an open point-cofinite cover of $X$ such that $G=\bigcap_{n} U_{n}$. For each $n$, let $U_{n}=\bigcup_{m} C_{m}^{n}$, a union of disjoint clopen sets. Define $\Psi: X \rightarrow \mathbb{E} \mathbb{F}$ by

$$
\Psi(x)(n)= \begin{cases}m, & m \in \mathbb{N}, x \in C_{m}^{n}, \\ \infty, & x \notin U_{n} .\end{cases}
$$

As $\left\{U_{n}: n \in \mathbb{N}\right\}$ is a point-cofinite cover of $X, \Psi(x)$ is eventually finite for each $x \in X$.

$\Psi$ is continuous: A basic open set in $\mathbb{E F}$ has the form $\prod_{n} V_{n}$ such that there are finite $I_{0}, I_{1} \subseteq \mathbb{N}$ and elements $m_{n}, n \in I_{0} \cup I_{1}$, for which: For each $n \in I_{0}, V_{n}=\left\{m_{n}\right\}$, for each $n \in I_{1}, V_{n}=\left\{m_{n}, m_{n}+1, \ldots\right\} \cup\{\infty\}$, and for each $n \notin I_{0} \cup I_{1}, V_{n}=\mathbb{N} \cup\{\infty\}$. Now,

$$
\Psi^{-1}\left[\prod_{n \in \mathbb{N}} V_{n}\right]=\bigcap_{n \in I_{0}} C_{m_{n}}^{n} \cap \bigcap_{n \in I_{1}}\left(X \backslash\left(\bigcup_{k<m_{n}} C_{k}^{n}\right)\right)
$$

is open.

Thus, $\Psi[X]$ is bounded by some $g \in \mathbb{N}^{\mathbb{N}}$. Now,

$$
G=\{x \in X:(\forall n) \Psi(x)(n)<\infty\}=\Psi^{-1}\left[\left\{f \in \mathbb{N}^{\mathbb{N}}: f \leq^{*} g\right\}\right] .
$$

The set $\left\{f \in \mathbb{N}^{\mathbb{N}}: f \leq^{*} g\right\}$ is an $F_{\sigma}$ subset of $\mathbb{E F}$. Indeed, let $\left\{g_{n}: n \in \mathbb{N}\right\}$ enumerate all elements of $\mathbb{N}^{\mathbb{N}}$ which are eventually equal to $g$. Then $\left\{f \in \mathbb{N}^{\mathbb{N}}: f \leq^{*} g\right\}=$ 
$\bigcup_{n}\left\{f \in \mathbb{E} \mathbb{F}: f \leq g_{n}\right\}$. Thus, $G$ is an $F_{\sigma}$ subset of $X$. As $\bigcup_{\text {fin }}(\mathrm{O}, \Gamma)$ is hereditary for closed subsets and preserved by countable unions, $G$ satisfies $\mathrm{U}_{\text {fin }}(\mathrm{O}, \Gamma)$.

Recall that a topological space $X$ is a $\sigma$ space if each $G_{\delta}$ subset of $X$ is an $F_{\sigma}$ subset of $X$. The proof of Theorem 2 actually shows that $(2) \Rightarrow(3),(2) \Rightarrow(1),(3) \Rightarrow(4)$, and (4) $\Rightarrow(2)$ in the following theorem (and therefore establishes it).

Theorem 5. The following are equivalent:

(1) $X$ is hereditarily $\mathrm{U}_{\text {fin }}(\mathrm{O}, \Gamma)$.

(2) Each $G_{\delta}$ subset of $X$ satisfies $U_{\text {fin }}(O, \Gamma)$.

(3) Every continuous image of $X$ in $\mathbb{E} \mathbb{F}$ is bounded.

(4) $X$ satisfies $\mathrm{U}_{\text {fin }}(\mathrm{O}, \Gamma)$ and is a $\sigma$ space.

The implication $(1) \Rightarrow(4)$ in Theorem 5 was first proved by Fremlin and Miller [15]. The implication $(4) \Rightarrow(1)$ can be alternatively deduced from Theorem 3.12 of [11] and Corollary 10 of [8]. An additional equivalent formulation was discovered by Sakai. Recall the definition of $\sigma^{\prime}$ space from the introduction (page 354).

Theorem 6 (Sakai). Let $X \subseteq \mathbb{R}$. Then $X$ is a $\sigma^{\prime}$ space if, and only if, $X$ is hereditarily $\mathrm{U}_{\text {fin }}(\mathrm{O}, \Gamma)$.

Proof. This follows from Theorem 5.7 of [22]: $X$ satisfies $\mathrm{U}_{\text {fin }}(\mathrm{O}, \Gamma)$ if, and only if, for each $G_{\delta}$ set $G \subseteq \mathbb{R}$ containing $X$, there is an $F_{\sigma}$ set $F \subseteq \mathbb{R}$ such that $X \subseteq F \subseteq G$.

$(\Rightarrow)$ As being a $\sigma^{\prime}$ space is hereditary, it suffices to show that $X$ satisfies $\bar{U}_{\text {fin }}(\mathrm{O}, \Gamma)$. Indeed, for each $G_{\delta}$ set $G \subseteq \mathbb{R}$ containing $X$, let $E=\mathbb{R} \backslash G$, and take an $F_{\sigma}$ set $F \subseteq \mathbb{R}$ disjoint from $E$ such that $X \subseteq E \cup F$. Then $X \subseteq F \subseteq G$.

$(\Leftarrow)$ Let $E \subseteq \mathbb{R}$ be $F_{\sigma}$. As $X \backslash E$ satisfies $\mathrm{U}_{\text {fin }}(\mathrm{O}, \Gamma)$ and is a subset of the $G_{\delta}$ set $\mathbb{R} \backslash E$, there is an $F_{\sigma}$ set $F \subseteq \mathbb{R}$ such that $X \backslash E \subseteq F \subseteq \mathbb{R} \backslash E$. Then $E \cap F=\emptyset$ and $X \subseteq E \cup F$.

To indicate the potential usefulness of Theorem 2, we use it to give slightly more direct proofs of two known theorems. Recall the definition of QN spaces from the introduction (page 355).

Theorem 7 (Recław [30]). If $X$ is a $Q N$ space, then $X$ is hereditarily $\mathrm{U}_{\mathrm{fin}}(\mathrm{O}, \Gamma)$.

Proof. Let $Y \subseteq \mathbb{E F}$ be a continuous image of $X$. Then $Y$ is a QN space. By Theorem 2, it suffices to show that $Y$ is bounded. For each $n$, and each $y \in Y$, define

$$
f_{n}(y)=\frac{1}{\min y^{-1}(n)},
$$

using the natural conventions that $\min \emptyset=\infty$ and $1 / \infty=0$. Then $\lim _{n} f_{n}(y)=0$ for all $y \in Y$. As $Y$ is a $\mathrm{QN}$ space, there are positive $\epsilon_{n}, n \in \mathbb{N}$, dominating this convergence. For each $k$, let

$$
Y_{k}=\left\{y \in Y:(\forall n \geq k) f_{n}(y)<\epsilon_{n}\right\} .
$$

Then $Y=\bigcup_{k} Y_{k}$. We will show that each $Y_{k}$ is bounded. 
Fix $k$. Take an increasing $g \in \mathbb{N}^{\mathbb{N}}$ such that $g(1)=k$ and for each $n, \epsilon_{m}<1 / n$ for all $m \geq g(n)$. Then $Y_{k}$ is bounded by $g$. Let $y \in Y_{k}$. Fix $n$ such that $y(n)<\infty$. If $y(n) \leq k$, then $y(n) \leq g(1) \leq g(n)$. Otherwise, $y(n)>k$, and since $y \in Y_{k}, f_{y(n)}(y)<\epsilon_{y(n)}$. Thus,

$$
\frac{1}{n} \leq \frac{1}{\min y^{-1}(y(n))}=f_{y(n)}(y)<\epsilon_{y(n)},
$$

and therefore $y(n)$ cannot be greater than $g(n)$.

Theorem 8 (Recław [30]). If $X$ is a $Q N$ space, then $X$ is a $\sigma$ space.

Proof. Apply Theorems 5 and 7.

The following sections give a deeper reason for the last two theorems.

\section{Bounded Borel images}

Our main goal in this section is to establish the equivalence in the following Theorem 9. The implication $(2) \Rightarrow(1)$ in this theorem is Proposition 9 of Scheepers [36]. The implication $(1) \Rightarrow(2)$ is the more difficult one, and will be proved in a sequence of related results.

Theorem 9. The following are equivalent:

(1) $C_{p}(X)$ is an $\alpha_{1}$ space.

(2) Each Borel image of $X$ in $\mathbb{N}^{\mathbb{N}}$ is bounded.

Proof. (2) $\Rightarrow$ (1) Consider a sheaf $\left\{A_{n}: n \in \mathbb{N}\right\}$ at $f \in C_{p}(X)$. For each $n$, enumerate $A_{n}=\left\{f_{m}^{n}: m \in \mathbb{N}\right\}$ bijectively. Define a Borel function $\Psi: X \rightarrow \mathbb{N}^{\mathbb{N}}$ by

$$
\Psi(x)(n)=\min \left\{k:(\forall m \geq k)\left|f_{m}^{n}(x)-f(x)\right| \leq 1 / n\right\} .
$$

Let $g \in \mathbb{N}^{\mathbb{N}}$ bound $\Psi[X]$, and take the amalgamation $B=\bigcup_{n}\left\{f_{m}^{n}: m \geq g(n)\right\}$. Then $B$ converges to $f$.

$(1) \Rightarrow(2)$ Assume that $C_{p}(X)$ is an $\alpha_{1}$ space. Then the subspace $C_{p}(X,\{0,1\})$ of $C_{p}(X)$, consisting of all continuous functions $f: X \rightarrow\{0,1\}$, is an $\alpha_{1}$ space. ${ }^{4}$ Each element of $C_{p}(X,\{0,1\})$ has the form $\chi_{U}$, the characteristic function of a clopen set $U \subseteq X$. Immediately from the definition, a sequence $\chi_{U_{n}}$ of elements of $C_{p}(X,\{0,1\})$ converges pointwise to the constant function 1 if, and only if, $\left\{U_{n}: n \in \mathbb{N}\right\}$ is a clopen point-cofinite cover of $X$. This gives the following, which is due to Bukovský-Haleš (cf. [9, Theorem 17]), and independently Sakai (cf. [33, Theorem 3.7]).

Lemma 10. The following are equivalent:

(1) $C_{p}(X,\{0,1\})$ is an $\alpha_{1}$ space.

(2) For each family $\left\{\mathcal{U}_{n}: n \in \mathbb{N}\right\}$ of pairwise disjoint clopen point-cofinite covers of $X$, there are cofinite $\mathcal{V}_{n} \subseteq \mathcal{U}_{n}, n \in \mathbb{N}$, such that $\bigcup_{n} \mathcal{V}_{n}$ is a point-cofinite cover of $X$

\footnotetext{
4 In fact, by the methods of Gerlits-Nagy [18], the converse implication also holds. This fact will not be used in our proof.
} 
A function $f$ with domain $X$ is a discrete limit of functions $f_{n}, n \in \mathbb{N}$, if for each $x \in X$, $f_{n}(x)=f(x)$ for all but finitely many $n$.

Each bijectively enumerated family $\mathcal{U}=\left\{U_{n}: n \in \mathbb{N}\right\}$ of subsets of a set $X$ induces a Marczewski map $\mathcal{U}: X \rightarrow P(\mathbb{N})$ defined by

$$
\mathcal{U}(x)=\left\{n \in \mathbb{N}: x \in U_{n}\right\}
$$

for each $x \in X$. The main step in our proof is the following.

Lemma 11. Assume that $C_{p}(X,\{0,1\})$ is an $\alpha_{1}$ space, and $\mathcal{U}=\left\{U_{n}: n \in \mathbb{N}\right\}$ is a bijectively enumerated family of open subsets of $X$. Then the Marczewski map $\mathcal{U}: X \rightarrow$ $P(\mathbb{N})$ is a discrete limit of continuous functions.

Proof. First, consider the case where for each $n, U_{n}$ is not clopen.

For each $n$, write $U_{n}$ as a union $\bigcup_{m} C_{m}^{n}$ of nonempty disjoint clopen sets. We may assume that the partitions are disjoint: Inductively, for each $n=2,3, \ldots$, consider the elements $C_{m}^{n}, m \in \mathbb{N}$, of the $n$th partition. For each $m$, if $C_{m}^{n}$ appears in the partition of $U_{k}$ for some $k<n$, merge (in the $n$th partition) $C_{m}^{n}$ with some other element of the $n$th partition. Continue in this manner until the $n$th partition is disjoint from all previous partitions.

Thus, the families $\mathcal{U}_{n}=\left\{\left(C_{m}^{n}\right)^{\mathrm{C}}: m \in \mathbb{N}\right\}$ are disjoint clopen point-cofinite covers of $X$. By Lemma 10, there are $k_{n}, n \in \mathbb{N}$, and subsets $\mathcal{V}_{n}=\left\{\left(C_{m}^{n}\right)^{\mathrm{C}}: m \geq k_{n}\right\} \subseteq \mathcal{U}_{n}$, $n \in \mathbb{N}$, such that $\bigcup_{n} \mathcal{V}_{n}$ is a point-cofinite cover of $X$. In other words,

$$
\mathcal{V}=\left\{\bigcap_{m=k_{n}}^{\infty}\left(C_{m}^{n}\right)^{\mathrm{C}}: n \in \mathbb{N}\right\}
$$

is a point-cofinite cover of $X$.

For each $n, m$, let

$$
U_{m}^{n}=\bigcup_{i=1}^{\max \left\{m, k_{n}\right\}} C_{i}^{n} .
$$

For each $m$, define $\Psi_{m}: X \rightarrow P(\mathbb{N})$ by

$$
\Psi_{m}(x)=\left\{n: x \in U_{m}^{n}\right\} .
$$

As each $U_{m}^{n}$ is clopen, $\Psi_{m}$ is continuous. It remains to prove that, viewed as a Marczewski map, $\mathcal{U}$ is a discrete limit of the maps $\Psi_{m}, m \in \mathbb{N}$.

Fix $x \in X$. Let $N$ be such that $x \in \bigcap_{m=k_{n}}^{\infty}\left(C_{m}^{n}\right)^{\mathrm{C}}$ for all $n \geq N$. For each $n<N$ with $x \in U_{n}$, let $m_{n}$ be such that $x \in U_{m_{n}}^{n}$. Set $M=\max \left\{m_{n}: n<N\right\}$.

Fix $m \geq M$. We show that $x \in U_{m}^{n}$ if, and only if, $x \in U_{n}$. One direction follows from $U_{m}^{n} \subseteq U_{n}$. To prove the other direction, assume that $x \in U_{n}$, and consider the two possible cases: If $n<N$, then $x \in U_{m}^{n}$ because $m \geq M \geq m_{n}$, and we are done. Thus, assume that $n \geq N$. Then $x \in \bigcap_{i=k_{n}}^{\infty}\left(C_{i}^{n}\right)^{\mathrm{C}}$. As $x \in U_{n}=\bigcup_{m} C_{m}^{n}$, it follows that $x \in \bigcup_{i=1}^{k_{n}-1} C_{i}^{n} \subseteq U_{m}^{n}$

Thus, for each $x \in X$ there is $M$ such that $\Psi_{m}(x)=\mathcal{U}(x)$ for all $m \geq M$. This completes the proof in the case that no $U_{n}$ is clopen. 
For the remaining case, let $I \subseteq \mathbb{N}$ be the set of all $n$ such that $U_{n}$ is not clopen. The previous case shows that $\mathcal{U}_{I}=\left\{U_{n}: n \in I\right\}$, viewed as a Marczewski function from $X$ to $P(I)$, is a discrete limit of continuous functions $\Psi_{m}: X \rightarrow P(I)$

For each $m$, define $\Phi_{m}: X \rightarrow P(\mathbb{N})$ by

$$
\Phi_{m}(x)=\left\{n:\left(n \in I \text { and } n \in \Psi_{m}(x)\right) \text { or }\left(n \notin I \text { and } x \in U_{n}\right)\right\} .
$$

Then $\mathcal{U}$ is a discrete limit of the continuous functions $\Phi_{m}, m \in \mathbb{N}$.

As $X$ satisfies item (2) of Lemma 10, it satisfies $U_{\text {fin }}(O, \Gamma)$ : Refine each given cover to a clopen cover, turn it to a clopen point-cofinite cover by taking finite unions, and make the point-cofinite covers disjoint.

Assume that $\mathcal{U}$ is a countable family of open subsets of $X$. By Lemma 11, the Marczewski map $\mathcal{U}: X \rightarrow P(\mathbb{N})$ is a discrete limit of continuous functions $\Psi_{n}$.

Clearly, every discrete limit is a quasi-normal limit. The proof of [10, Theorem 4.8] actually establishes the following.

Lemma 12. Assume that $P$ is a property of topological spaces, which is preserved by taking closed subsets, continuous images and countable unions. If $X$ has the property $P$ and $\Psi: X \rightarrow Y$ is a quasi-normal limit of continuous functions into a metric space $Y$, then $\Psi[X]$ has the property $P$.

Proof. Let $\Psi_{n}, n \in \mathbb{N}$, be continuous functions as in the premise of the lemma, and let $\epsilon_{n}$, $n \in \mathbb{N}$, be as in the definition of quasi-normal convergence. For each $k$,

$$
X_{k}=\left\{x \in X:(\forall n, m \geq k) d\left(\Psi_{n}(x), \Psi_{m}(x)\right) \leq \epsilon_{n}+\epsilon_{m}\right\}
$$

is a closed subset of $X$, and the functions $\Psi_{n}$ converge to $\Psi$ uniformly on $X_{k}$. Thus, $\Psi$ is continuous on $X_{k}$, and therefore $\Psi\left[X_{k}\right]$ has the property $P$.

Now, $X=\bigcup_{k} X_{k}$, and therefore $\Psi[X]=\bigcup_{k} \Psi\left[X_{k}\right]$ has the property $P$.

It follows that for each countable family $\mathcal{U}$ of open subsets of $X, \mathcal{U}[X]$ satisfies $\bigcup_{\text {fin }}(\mathrm{O}, \Gamma)$.

Let $\mathrm{F}, \mathrm{B}$ denote the families of all countable closed and all countable Borel covers of $X$, respectively. Similarly, let $\mathrm{F}_{\Gamma}, \mathrm{B}_{\Gamma}$ denote the families of all countable closed and all Borel point-cofinite covers of $X$. Following is a striking result of Bukovský, Recław, and Repický [10]. In their terminology, it tells that the family of closed subsets of $X$ is weakly distributive if, and only if, so is the family of Borel subsets of $X$. In the language of selection principles, this result has the following compact form.

Lemma 13 (Bukovský-Recław-Repický [10]). $\mathrm{U}_{\text {fin }}\left(\mathrm{F}, \mathrm{F}_{\Gamma}\right)=\mathrm{U}_{\text {fin }}\left(\mathrm{B}, \mathrm{B}_{\Gamma}\right)$.

Proof. Assume that $X$ satisfies $\mathrm{U}_{\mathrm{fin}}\left(\mathrm{F}, \mathrm{F}_{\Gamma}\right)$. We first show that $X$ is a $\sigma$ space $[10$, Theorem 5.2].

Assume that $G=\bigcap_{n} U_{n}$ where for each $n, U_{n} \supseteq U_{n+1}$ are open subsets of $X$. Write, for each $n$,

$$
U_{n}=\bigcup_{m \in \mathbb{N}} C_{m}^{n},
$$

where for each $m, C_{m}^{n} \subseteq C_{m+1}^{n}$ are closed subsets of $X$. We may assume that the closed 
cover $\left\{C_{m}^{n} \cup\left(X \backslash U_{n}\right): m \in \mathbb{N}\right\}$ of $X$ has no finite subcover. ${ }^{5}$ As $X$ satisfies $\cup_{\text {fin }}\left(\mathrm{F}, \mathrm{F}_{\Gamma}\right)$ and each given cover is monotone, there are $m_{n}, n \in \mathbb{N}$, such that $\left\{C_{m_{n}}^{n} \cup\left(X \backslash U_{n}\right): n \in \mathbb{N}\right\}$ is a closed point-cofinite cover of $X$. For each $k$ define

$$
Z_{k}=\bigcap_{n=k}^{\infty} C_{m_{n}}^{n} .
$$

Then each $Z_{k}$ is a closed subset of $X$, and $G=\bigcup_{k} Z_{k}$ is $F_{\sigma}$. This shows that $X$ is a $\sigma$ space.

Now, assume that $\mathcal{U}_{n} \in \mathrm{B}, n \in \mathbb{N}$. Then for each $n$, each element of $\mathcal{U}_{n}$ is $F_{\sigma}$ and can therefore be replaced by countably many closed sets. Applying $\mathrm{U}_{\text {fin }}\left(\mathrm{F}, \mathrm{F}_{\Gamma}\right)$ to the thus modified covers, we obtain a cover in $\mathrm{F}_{\Gamma}$. For each $n$, extend each of the finitely many chosen elements of the $n$th cover to an $F_{\sigma}$ set from the original cover $\mathcal{U}_{n}$, to obtain an element of $\mathrm{B}_{\Gamma}$ chosen in accordance with the definition of $\mathrm{U}_{\text {fin }}\left(\mathrm{B}, \mathrm{B}_{\Gamma}\right){ }^{6}$

Lemma 14. The following are equivalent:

(1) X satisfies $\mathrm{U}_{\text {fin }}\left(\mathrm{B}, \mathrm{B}_{\Gamma}\right)$.

(2) For each countable family $\mathcal{U}$ of open subsets of $X, \mathcal{U}[X]$ satisfies $\bigcup_{\text {fin }}(\mathrm{O}, \Gamma)$.

(3) For each countable family $\mathcal{C}$ of closed subsets of $X, \mathcal{C}[X]$ satisfies $U_{\text {fin }}(O, \Gamma)$.

Proof. $(2) \Leftrightarrow(3)$ Use the auto-homeomorphism of $P(\mathbb{N})$ defined by mapping a set to its complement.

$(1) \Rightarrow(2)$ The Marczewski map $\mathcal{U}: X \rightarrow P(\mathbb{N})$ is Borel. It is easy to see that $\mathrm{U}_{\text {fin }}\left(\mathrm{B}, \mathrm{B}_{\Gamma}\right)$ is preserved by Borel images [39]. Thus, $\mathcal{U}[X]$ satisfies $\mathrm{U}_{\text {fin }}\left(\mathrm{B}, \mathrm{B}_{\Gamma}\right)$, and in particular $\mathrm{U}_{\text {fin }}(\mathrm{O}, \Gamma)$.

(3) $\Rightarrow$ (1) By Lemma 13, it suffices to show that $X$ satisfies $U_{\text {fin }}\left(\mathrm{F}, \mathrm{F}_{\Gamma}\right)$. For each $\mathcal{C}=$ $\left\{C_{n}: n \in \mathbb{N}\right\} \in \mathrm{F}$ which does not contain a finite subcover, $\left\{\bigcup_{m \leq n} C_{m}: n \in \mathbb{N}\right\} \in \mathrm{F}_{\Gamma}$. Thus, $\mathrm{U}_{\text {fin }}\left(\mathrm{F}, \mathrm{F}_{\Gamma}\right)=\mathrm{U}_{\text {fin }}\left(\mathrm{F}_{\Gamma}, \mathrm{F}_{\Gamma}\right){ }^{7}$ and we prove the latter property.

Let $\mathcal{C}_{n}=\left\{C_{m}^{n}: m \in \mathbb{N}\right\}, n \in \mathbb{N}$, be bijectively enumerated closed point-cofinite covers of $X$ which do not contain finite subcovers. We may assume that these covers are pairwise disjoint [35].

Let $\mathcal{C}=\bigcup_{n} \mathcal{C}_{n}$, and consider the Marczewski map $\mathcal{C}: X \rightarrow P(\mathbb{N} \times \mathbb{N})$ defined by

$$
\mathcal{C}(x)=\left\{(n, m): x \in C_{m}^{n}\right\}
$$

for all $x \in X$. For each $(n, m), O_{(n, m)}=\{A \subseteq \mathbb{N} \times \mathbb{N}:(n, m) \in A\}$ is an open subset of $P(\mathbb{N} \times \mathbb{N})$, and for each $n, \mathcal{U}_{n}=\left\{O_{(n, m)}: m \in \mathbb{N}\right\}$ is an open cover of $\mathcal{C}[X]$ which does not contain a finite subcover. As $\mathcal{C}[X]$ satisfies $\mathrm{U}_{\text {fin }}(\mathrm{O}, \Gamma)$, there are $k_{n}, n \in \mathbb{N}$, such that $\left\{\bigcup_{m<k_{n}} O_{(n, m)}: n \in \mathbb{N}\right\}$ is a point-cofinite cover of $\mathcal{C}[X]$. Then $\left\{\bigcup_{m<k_{n}} C_{m}^{n}: n \in \mathbb{N}\right\}$ is a point-cofinite cover of $X$ (it is infinite because $X$ does not appear there as an element). ${ }^{8}$

\footnotetext{
5 If there are infinitely many $n$ for which there is some $m_{n}$ with $C_{m_{n}}^{n}=U_{n}$, then $G=\bigcap_{n} C_{m_{n}}^{n}$ is closed and we are done. Otherwise, we can ignore finitely many $n$ and assume that there are no $n, m$ such that $C_{m}^{n}=U_{n}$ contains $G$.

6 This argument, in more general form, appears in [11, Theorem 2.1].

7 This statement holds in a more general form [22].

8 The argument is standard: For each finite family of proper subsets of $X$, there is a finite subset of $X$ not contained in any member of this family.
} 
By Lemma $14, X$ satisfies $\mathrm{U}_{\text {fin }}\left(\mathrm{B}, \mathrm{B}_{\Gamma}\right)$. It remains to observe the following. For the reader's convenience, we reproduce the proof of the implication needed in the present proof.

Lemma 15 (Bartoszyński-Scheepers [4]). X satisfies $\mathrm{U}_{\mathrm{fin}}\left(\mathrm{B}, \mathrm{B}_{\Gamma}\right)$ if, and only if, each Borel image of $X$ in $\mathbb{N}^{\mathbb{N}}$ is bounded.

Proof. $(\Rightarrow)$ Assume that $Y \subseteq \mathbb{N}^{\mathbb{N}}$ is a Borel image of $X$. Then $Y$ satisfies $\mathrm{U}_{\text {fin }}\left(\mathrm{B}, \mathrm{B}_{\Gamma}\right)$. By taking the image of $Y$ under the continuous mapping $f(n) \mapsto f(1)+\cdots+f(n)$ defined on $\mathbb{N}^{\mathbb{N}}$, we may assume that all elements in $Y$ are nondecreasing.

We first consider the uninteresting case: There is an infinite $I \subseteq \mathbb{N}$ such that for each $n \in I, F_{n}=\{f(n): f \in Y\}$ is finite. For each $n$, let $m \in I$ be minimal such that $n \leq m$, and define $g(n)=\max F_{m}$. Then $Y$ is bounded by $g$.

Thus, assume that there is $N$ such that for each $n \geq N,\{f(n): f \in Y\}$ is infinite. For all $n, m$, consider the open set $U_{m}^{n}=\{f \in Y: f(n) \leq m\}$. Then for each $n \geq N$, $\mathcal{U}_{n}=\left\{U_{m}^{n}: m \in \mathbb{N}\right\}$ is an open point-cofinite cover of $Y$. Apply $\mathrm{U}_{\text {fin }}\left(\mathrm{B}, \mathrm{B}_{\Gamma}\right)$ to obtain for each $n \geq N$ a finite set $F_{n} \subseteq \mathbb{N}$ such that $\left\{\bigcup_{m \in F_{n}} U_{m}^{n}: n \in \mathbb{N}\right\}$ is a point-cofinite cover of $Y$. Define $g \in \mathbb{N}^{\mathbb{N}}$ by $g(n)=\max F_{n}$ for each $n \geq N$ (and arbitrary for $n<N$ ). Then $Y$ is bounded by $g$.

This completes the proof of Theorem 9 .

Remark 16. Let $A \subseteq^{*} B$ mean that $A \backslash B$ is finite. A semifilter is a family $\mathcal{F}$ of infinite subsets of $\mathbb{N}$ such that for each $A \in \mathcal{F}$ and each $B \subseteq \mathbb{N}$ with $A \subseteq \subseteq^{*} B$, we have $B \in \mathcal{F}$. In [43] it is proved that if in item (2) of Lemma 14 we replace $\mathcal{U}[X]$ with the semifilter it generates, then we obtain a characterization of $U_{f i n}(O, \Gamma)$. Lemma 14 shows that moving to the generated semifilter is essential to obtain this result, since $U_{\text {fin }}\left(B, B_{\Gamma}\right)$ is strictly stronger than $\mathrm{U}_{\text {fin }}(\mathrm{O}, \Gamma)$.

\section{Applications}

\subsection{QN spaces}

We begin with a straightforward proof of one implication in the following theorem (which answers in the affirmative Problem 2 of Scheepers [36]). Because of the importance of this result, we also supply a proof for the other implication.

Theorem 17 (Sakai [33], Bukovský-Haleš [9]). $X$ is a $Q N$ space if, and only if, $C_{p}(X)$ is an $\alpha_{1}$ space.

Proof. $(\Leftarrow)$ This is Theorem 4 of [36]. Using Theorem 9 this becomes straightforward: Assume that $C_{p}(X)$ is an $\alpha_{1}$ space. Given $f_{n}, n \in \mathbb{N}$, converging pointwise to 0 , define a Borel function $\Psi: X \rightarrow \mathbb{N}^{\mathbb{N}}$ by

$$
\Psi(x)(n)=\min \left\{k:(\forall m \geq k)\left|f_{m}(x)\right|<1 / n\right\} .
$$

By Theorem $9, \Psi[X]$ is bounded by some $g \in \mathbb{N}^{\mathbb{N}}$. For each $x \in X$ and all but finitely many $n,\left|f_{m}(x)\right|<1 / n$ for each $m \geq g(n)$. For each $n$ and each $m$ with $g(n) \leq m<$ $g(n+1)$, take $\epsilon_{m}=1 / n$. 
$(\Rightarrow)$ Assume that $X$ is a QN space, and $\left\{A_{n}: n \in \mathbb{N}\right\}$ is a countable sheaf at $f \in$ $C_{p}(X)$. We may assume that $f$ is the constant zero function, and that the image of each member of each $A_{n}$ is contained in the unit interval [0,1].

For each $n$, enumerate $A_{n}=\left\{f_{m}^{n}: m \in \mathbb{N}\right\}$ bijectively. For each $m$, define $g_{m} \in$ $C_{p}(X)$ by

$$
g_{m}(x)=\sup \left\{f_{m}^{n}(x) / n: n \in \mathbb{N}\right\}
$$

for all $x \in X$. Then $\left\{g_{m}: m \in \mathbb{N}\right\}$ converges pointwise to the zero function. As $X$ is a QN space, there are positive $\epsilon_{m}, m \in \mathbb{N}$, converging to 0 , such that $X$ is the increasing union of the sets

$$
X_{n}=\left\{x \in X:(\forall m \geq n) g_{m}(x) \leq \epsilon_{m}\right\} .
$$

For each $n$, choose $m_{n}$ such that $n \epsilon_{m} \leq 1 / n$ for all $m \geq m_{n}$. We claim that the amalgamation $B=\bigcup_{n}\left\{f_{m}^{n}: m \geq m_{n}\right\}$ converges pointwise to the zero function. Indeed, fix $x \in X$ and a positive $\epsilon$. Take $N$ such that $x$ belongs to $X_{N}$ (and thus to all $X_{k}$ with $k \geq N$ ) and such that $1 / N \leq \epsilon$. For each $n \geq N$ and each $m \geq m_{n}$,

$$
f_{m}^{n}(x) \leq n \cdot g_{m}(x) \leq n \epsilon_{m} \leq 1 / n \leq \epsilon .
$$

And for each $n<N$, there are only finitely many $m$ such that $f_{m}^{n}(x)>\epsilon$. Thus, for all but finitely many $f \in B, f(x) \leq \epsilon$.

A beautiful direct (but tricky) proof for $(\Leftarrow)$ of Theorem 17 was recently discovered by Bukovský [7].

Theorems 9 and 17 solve in the affirmative Problem 22 from [9].

Corollary 18. $X$ is a $Q N$ space if, and only if, each Borel image of $X$ in $\mathbb{N}^{\mathbb{N}}$ is bounded.

Theorem 19 (Recław [30]). The QN property is hereditary.

Proof. The property of having bounded Borel images in $\mathbb{N}^{\mathbb{N}}$ is hereditary.

Answering Question 5.8 of Shakhmatov [40] (attributed to Scheepers), Sakai [33] and independently Bukovský-Haleš [9] gave a characterization of the QN property in terms of covering properties of $X$. Their characterization uses the new Kočinac $\alpha_{1}$ selection principle [25]. Theorem 15 and Corollary 18 give a new characterization in terms of the classical Hurewicz selection principle: $U_{\text {fin }}\left(B, B_{\Gamma}\right)$. This selection hypothesis can be stated in a more elegant manner. For families of covers $\mathscr{A}, \mathscr{B}$ of $X$, define

$\mathrm{S}_{1}(\mathscr{A}, \mathscr{B}):$ Whenever $\mathcal{U}_{1}, \mathcal{U}_{2}, \ldots \in \mathscr{A}$, there exist elements $U_{n} \in \mathcal{U}_{n}, n \in \mathbb{N}$, such that $\left\{U_{n}: n \in \mathbb{N}\right\} \in \mathscr{B}$.

Then $\mathrm{U}_{\text {fin }}\left(\mathrm{B}, \mathrm{B}_{\Gamma}\right)=\mathrm{S}_{1}\left(\mathrm{~B}_{\Gamma}, \mathrm{B}_{\Gamma}\right)$ [39]. By Lemma 13, also $\mathrm{U}_{\text {fin }}\left(\mathrm{F}, \mathrm{F}_{\Gamma}\right)=\mathrm{S}_{1}\left(\mathrm{~F}_{\Gamma}, \mathrm{F}_{\Gamma}\right)$. (This can also be proved directly.) We obtain the following new characterizations.

Corollary 20. The following are equivalent:

(1) $C_{p}(X)$ is an $\alpha_{1}$ space.

(2) $X$ is a $Q N$ space.

(3) $X$ satisfies $\mathrm{S}_{1}\left(\mathrm{~F}_{\Gamma}, \mathrm{F}_{\Gamma}\right)$.

(4) X satisfies $\mathrm{S}_{1}\left(\mathrm{~B}_{\Gamma}, \mathrm{B}_{\Gamma}\right)$. 


\subsection{Convergent sequences of Borel functions}

Let $B_{p}(X)$ be the space of all Borel real-valued functions on $X$, with the topology of pointwise convergence. We obtain the surprising result that if $C_{p}(X)$ is an $\alpha_{1}$ space, then so is $B_{p}(X)$. This is not provably the case for Arhangel'skiır's properties $\alpha_{2}, \alpha_{3}$, and $\alpha_{4}$. A topological space $Y$ is an $\alpha_{2}$ space if it satisfies $S_{1}\left(\Gamma_{y}, \Gamma_{y}\right)$ for each $y \in Y$, where $\Gamma_{y}$ is the family of all sequences converging to $y$. For the definitions of $\alpha_{3}$ and $\alpha_{4}$, see e.g. [36].

Corollary 21. The following are equivalent:

(1) Each Borel image of $X$ in $\mathbb{N}^{\mathbb{N}}$ is bounded.

(2) $C_{p}(X)$ is an $\alpha_{1}$ space.

(3) $B_{p}(X)$ is an $\alpha_{1}$ space.

(4) $B_{p}(X)$ is an $\alpha_{2}$ space.

(5) $B_{p}(X)$ is an $\alpha_{3}$ space.

(6) $B_{p}(X)$ is an $\alpha_{4}$ space.

Proof. (1) $\Rightarrow$ (3) This is proved verbatim as the proof of $(2) \Rightarrow(1)$ in Theorem 9.

$(3) \Rightarrow(2)$ is evident.

$(2) \Rightarrow(1)$ is due to the above mentioned result of Scheepers, and the equivalence of being a QN space and (1).

$(4) \Leftrightarrow(5) \Leftrightarrow(6)$ is proved as in Gerlits-Nagy [18] or Scheepers [36] (in fact, the Borel case is easier).

(3) $\Rightarrow(4)$ is evident.

(4) $\Rightarrow$ (1) It suffices to show that $X$ satisfies $\mathrm{S}_{1}\left(\mathrm{~B}_{\Gamma}, \mathrm{B}_{\Gamma}\right)$. Given $\mathcal{U}_{n} \in \mathrm{B}_{\Gamma}, n \in \mathbb{N}$, we find that for each $n, A_{n}=\left\{\chi_{U}: U \in \mathcal{U}_{n}\right\} \subseteq B_{p}(X)$ converges pointwise to 0 . Applying $\alpha_{2}$, let $U_{n} \in \mathcal{U}_{n}, n \in \mathbb{N}$, be such that $\chi_{U_{n}}$ converges pointwise to 0 . Then $\left\{U_{n}: n \in \mathbb{N}\right\}$ is a point-cofinite cover of $X$.

\subsection{Almost continuous functions}

A function $f: X \rightarrow Y$ is almost continuous [3] if for each nonempty $A \subseteq X$, the restriction of $f$ to $A$ has a point of continuity. $A C_{p}(X)$ is the space of all almost continuous real-valued functions on $X$, with the topology of pointwise convergence [5].

If $X$ and $Y$ are Tychonoff and $f: X \rightarrow Y$ is almost continuous, then for each $A \subseteq X$ the set of points of continuity of the restriction of $f$ to $A$ is open dense in $A$ [5]. Each function with the latter property is Borel [42]. Thus, $C_{p}(X) \subseteq A C_{p}(X) \subseteq B_{p}(X)$.

Corollary 22. $A C_{p}(X)$ is an $\alpha_{1}$ space if, and only if, $C_{p}(X)$ is an $\alpha_{1}$ space.

\section{4. $w Q N$ spaces and the Scheepers Conjecture}

$X$ is a $w Q N$ space [10] if each sequence of continuous real-valued functions on $X$ converging pointwise to zero has a subsequence converging to zero quasi-normally.

Two fundamental problems concerning wQN spaces appear in the literature: In [36, p. 269], [9, Problem 23], and [6, Problems 10.3-10.4], we are asked whether, consistently, 
every wQN space is a QN space. The Scheepers Conjecture [37] asserts that $X$ is a wQN space if, and only if, $X$ satisfies $S_{1}(\Gamma, \Gamma)$. It is still open whether the Scheepers Conjecture is provable. A striking result of Dow gives a positive answer to the first problem, and a consistently positive answer to the second.

Theorem 23 (Dow [14]). In the Laver model, each $\alpha_{2}$ space is an $\alpha_{1}$ space.

Let $\mathrm{C}_{\Gamma}$ denote the family of all clopen point-cofinite covers of $X$. Clearly, $\mathrm{S}_{1}(\Gamma, \Gamma)$ implies $\mathrm{S}_{1}\left(\mathrm{C}_{\Gamma}, \mathrm{C}_{\Gamma}\right)$.

Corollary 24. In the Laver model:

(1) $\mathrm{S}_{1}\left(\mathrm{~B}_{\Gamma}, \mathrm{B}_{\Gamma}\right)=\mathrm{S}_{1}\left(\mathrm{C}_{\Gamma}, \mathrm{C}_{\Gamma}\right)$.

(2) $X$ is a wQN space if, and only if, $X$ is a $Q N$ space.

(3) The Scheepers Conjecture holds.

In particular, these assertions are (simultaneously) consistent.

Proof. (1) Using the correspondence described just before Lemma 10, we find that $C_{p}(X)$ is an $\alpha_{2}$ space if, and only if, $X$ satisfies $\mathrm{S}_{1}\left(\mathrm{C}_{\Gamma}, \mathrm{C}_{\Gamma}\right)$. Thus, if $X$ satisfies $\mathrm{S}_{1}\left(\mathrm{C}_{\Gamma}, \mathrm{C}_{\Gamma}\right)$, then by Dow's Theorem 23, $C_{p}(X)$ is an $\alpha_{1}$ space. By Theorem $9, X$ satisfies $\mathrm{S}_{1}\left(\mathrm{~B}_{\Gamma}, \mathrm{B}_{\Gamma}\right)$.

(2) Assume that $X$ is a wQN space. Then $C_{p}(X)$ is an $\alpha_{2}$ space [34]. By Dow's Theorem 23, $C_{p}(X)$ is an $\alpha_{1}$ space. By Theorem $17, X$ is a QN space.

(3) $S_{1}(\Gamma, \Gamma)$ implies (in ZFC) being a wQN space [37]. Now, back in the Laver model, assume that $X$ is a wQN space. By (2), $X$ is a QN space. By Corollary 20, $X$ satisfies $\mathrm{S}_{1}\left(\mathrm{~B}_{\Gamma}, \mathrm{B}_{\Gamma}\right)$, and in particular $\mathrm{S}_{1}(\Gamma, \Gamma)$.

Remark 25. In [33,9] it is shown that $X$ is a wQN space if, and only if, $X$ satisfies $\mathrm{S}_{1}\left(\mathrm{C}_{\Gamma}, \mathrm{C}_{\Gamma}\right)$. Using this, (2) and (3) follow immediately from Corollary 24(1).

\section{5. $\overline{Q N}$ spaces and $M$ spaces}

$X$ is a $\overline{Q N}$ space [11] if each real-valued function (not necessarily continuous) on $X$ which is a pointwise limit of a sequence of continuous functions, is in fact a quasi-normal limit of those functions.

The following result is immediate from Theorem 9 and [11, Theorem 5.10(9)]. For completeness, we give a simple, direct proof.

Theorem 26. The following are equivalent:

(1) $X$ is a $\overline{Q N}$ space.

(2) $X$ is a $Q N$ space.

(3) Each sequence of Borel functions converging pointwise to 0, converges to 0 quasinormally.

(4) Each sequence of Borel functions converging pointwise to any function converges quasi-normally to this function.

Proof. $(1) \Rightarrow(2)$ is immediate.

$(2) \Rightarrow(3)$ Assume (2). By Theorem 9, each Borel image of $X$ in $\mathbb{N}^{\mathbb{N}}$ is bounded. Thus, an argument verbatim as in our proof of $(\Leftarrow)$ of Theorem 9 gives (3).

$(3) \Rightarrow(4)$ The limit function $f$ is also Borel, and $f_{n}-f$ converges to 0 .

$(4) \Rightarrow(1)$ is immediate. 
This shows that the first assumption in [11, Theorem 5.10(9)] is not needed. It also answers [11, Problem 6.11] in the affirmative. Based on [11, Theorem 6.9] and improving it, we also obtain the following solution of [11, Problem 6.10].

Corollary 27. Every QN space is an $M$ space.

The definition of $M$ space is available in [11].

4.6. $w Q N_{*}$ spaces

$X$ is a $w Q N_{*}$ space if each sequence of lower semi-continuous real-valued functions on $X$ converging pointwise to zero has a subsequence converging to zero quasi-normally. In his talk at the Third Workshop on Coverings, Selections, and Games in Topology (Serbia, April 2007), Bukovský defined $\mathrm{wQN}_{*}$ spaces and described his recent investigations of this property and its upper semi-continuous variant. The main problem he posed was: Is every QN space a $\mathrm{wQN}_{*}$ space?

Theorem 28. Every $Q N$ space is a $w Q N_{*}$ space.

Proof. Every lower semi-continuous function is Borel. Use Theorem 26.

Bukovský has later proved that the converse to Theorem 28 also holds [7], and therefore the notions coincide (with one another and with having bounded Borel images).

\subsection{Bounded-ideal convergence spaces}

The notion of ideal convergence originates in works of Steinhaus and Fast on statistical convergence, and was generalized by Bernstein, Katětov, and others (see [16] for an introduction). The following definitions are as in Jasinski-Recław [20]. Let $D$ be a countable set, and $\mathcal{I} \subseteq P(D)$ be an ideal (i.e., $\mathcal{I}$ contains all singletons and is closed under taking subsets and finite unions). Let $\mathcal{I}^{*}$ denote the filter $\{D \backslash A: A \in \mathcal{I}\}$ dual to $\mathcal{I}$. A sequence $\left\{r_{d}\right\}_{d \in D}$ of real numbers $\mathcal{I}$-converges to 0 if $\left\{d \in D:\left|r_{d}\right|<\epsilon\right\} \in \mathcal{I}^{*}$ for each positive $\epsilon$. A sequence $\left\{f_{d}\right\}_{d \in D}$ of continuous real-valued functions on $X \mathcal{I}$-converges to 0 if for each $x \in X$, the sequence $\left\{f_{d}(x)\right\}_{d \in D}$ of real numbers $\mathcal{I}$-converges to 0 . The space $X$ has the $\mathcal{I}$-convergence property if for each sequence $\left\{f_{d}\right\}_{d \in D}$ of continuous real-valued functions on $X$ which $\mathcal{I}$-converges to 0 , there is $A \in \mathcal{I}^{*}$ such that $\left\{f_{d}\right\}_{d \in A}$ converges pointwise to 0 .

We will use the following.

Lemma 29. In the definition of $\mathcal{I}$-convergence, it suffices to consider only sequences of distinct elements.

Proof. Let $\left\{f_{d}\right\}_{d \in D}$ be given. Enumerate $D=\left\{d_{n}: n \in \mathbb{N}\right\}$ bijectively. For each $n$, as the functions $f_{d_{n}}+1 / m, m \in \mathbb{N}$, are all distinct, there is $m\left(d_{n}\right) \in \mathbb{N}$ such that $m\left(d_{n}\right) \geq n$ and $f_{d_{n}}+1 / m\left(d_{n}\right) \notin\left\{f_{d_{1}}+1 / m\left(d_{1}\right), \ldots, f_{d_{n-1}}+1 / m\left(d_{n-1}\right)\right\}$.

It is easy to see that $\left\{f_{d}\right\}_{d \in D} \mathcal{I}$-converges to 0 if, and only if, $\left\{f_{d}+1 / m(d)\right\}_{d \in D}$ $\mathcal{I}$-converges to 0 . 
We use these definitions for $D=\mathbb{N} \times \mathbb{N}$. For $h \in \mathbb{N}^{\mathbb{N}}$, define $A_{h}=\{(n, m): m \leq h(n)\}$. The family $\left\{A_{h}: h \in \mathbb{N}^{\mathbb{N}}\right\}$ is closed under finite intersections, and generates the boundedideal

$$
\mathcal{I}_{b}=\left\{B \subseteq \mathbb{N} \times \mathbb{N}:\left(\exists h \in \mathbb{N}^{\mathbb{N}}\right) B \subseteq A_{h}\right\}
$$

$X$ has the bounded-ideal convergence property if it has the $\mathcal{I}_{b}$-convergence property.

The bounded-ideal, which is also the Fubini product $\emptyset \times$ Fin of the trivial ideal and the ideal of finite sets, plays a central role in studies of ideal convergence. For each analytic $P$-ideal $\mathcal{I}$, if any $X \subseteq \mathbb{R}$ not having Lebesgue measure zero has the $\mathcal{I}$-convergence property, then $\mathcal{I}$ is isomorphic to $\mathcal{I}_{b}$ [21]. For additional uses of this ideal and its associated convergence, see [16].

Jasinski and Recław [20] proved that every Sierpiński set has the bounded-ideal convergence property, and that if $X$ has the bounded-ideal convergence property, then $X$ is a $\sigma$ space. Both of these assertions follow at once from the following.

Theorem 30. The following are equivalent:

(1) $X$ has the bounded-ideal convergence property.

(2) $C_{p}(X)$ is an $\alpha_{1}$ space.

(3) Each Borel image of $X$ in $\mathbb{N}^{\mathbb{N}}$ is bounded.

Proof. By Theorem 9, it suffices to show that $(1) \Leftrightarrow(2)$.

(2) $\Rightarrow$ (1) Assume that $\left\{f_{(n, m)}\right\}_{(n, m) \in \mathbb{N} \times \mathbb{N}} \mathcal{I}_{b}$-converges to 0. By Lemma 29, we may assume that the elements $f_{(n, m)},(n, m) \in \mathbb{N} \times \mathbb{N}$, are distinct.

For each $x \in X$ and each positive $\epsilon,\left\{(n, m):\left|f_{(n, m)}(x)\right|<\epsilon\right\} \in \mathcal{I}_{b}^{*}$, that is, there is $h \in \mathbb{N}^{\mathbb{N}}$ such that $\left\{(n, m):\left|f_{(n, m)}(x)\right|<\epsilon\right\} \supseteq(\mathbb{N} \times \mathbb{N}) \backslash A_{h}$. Thus, $\left|f_{(n, m)}(x)\right|<\epsilon$ for all $n, m \in \mathbb{N}$ such that $h(n)<m$. It follows that for each $n,\left\{f_{(n, m)}\right\}_{m \in \mathbb{N}}$ converges pointwise to 0 .

As $C_{p}(X)$ is an $\alpha_{1}$ space, there is for each $n$ a number $h(n) \in \mathbb{N}$ such that $\left\{f_{(n, m)}\right.$ : $n, m \in \mathbb{N}, m>h(n)\}$ converges pointwise to 0 , and since the enumeration is bijective, the sequence $\left\{f_{(n, m)}\right\}_{(n, m) \in(\mathbb{N} \times \mathbb{N}) \backslash A_{h}}$ also converges pointwise to 0 . As $(\mathbb{N} \times \mathbb{N}) \backslash A_{h} \in \mathcal{I}_{b}^{*}$, this shows that $X$ has the bounded-ideal convergence property.

$(1) \Rightarrow(2)$ Assume that for each $n$ the sequence $\left\{f_{(n, m)}\right\}_{m \in \mathbb{N}}$ converges pointwise to 0 . For each $x \in X$, each positive $\epsilon$, and each $n$, there is $h(n) \in \mathbb{N}$ such that $\left|f_{(n, m)}(x)\right|<\epsilon$ for all $m>h(n)$. Thus, $\left\{(n, m):\left|f_{(n, m)}(x)\right|<\epsilon\right\} \supseteq(\mathbb{N} \times \mathbb{N}) \backslash A_{h}$, that is, $\left\{f_{(n, m)}\right\}_{(n, m) \in \mathbb{N} \times \mathbb{N}} \mathcal{I}_{b}$-converges to 0 .

The bounded-ideal convergence property implies that there is $h \in \mathbb{N}^{\mathbb{N}}$ such that $\left\{f_{(n, m)}\right\}_{(n, m) \in(\mathbb{N} \times \mathbb{N}) \backslash A_{h}}$ converges pointwise to 0 , and therefore so does the sheaf amalgamation $\left\{f_{(n, m)}: n, m \in \mathbb{N}, m>h(n)\right\}$ (which can be enumerated as a subsequence of

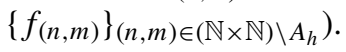

Corollary 31. The following are equivalent:

(1) X has the bounded-ideal convergence property.

(2) For each sequence $\left\{f_{d}\right\}_{d \in \mathbb{N} \times \mathbb{N}}$ of Borel real-valued functions on $X$ which $\mathcal{I}_{b}$-converges to 0 , there is $A \in \mathcal{I}_{b}^{*}$ such that $\left\{f_{d}\right\}_{d \in A}$ converges pointwise to 0 . 
(3) For each sequence $\left\{f_{d}\right\}_{d \in \mathbb{N} \times \mathbb{N}}$ of Borel real-valued functions on $X$ which $\mathcal{I}_{b}$-converges to a Borel function $f$, there is $A \in \mathcal{I}_{b}^{*}$ such that $\left\{f_{d}\right\}_{d \in A}$ converges pointwise to $f$.

(4) For each sequence $\left\{f_{d}\right\}_{d \in \mathbb{N} \times \mathbb{N}}$ of Borel real-valued functions on $X$ which $\mathcal{I}_{b}$-converges to a function $f$, there is $A \in \mathcal{I}_{b}^{*}$ such that $\left\{f_{d}\right\}_{d \in A}$ converges pointwise to $f$.

Proof. (1) $\Rightarrow(2)$ Replace "continuous" by "Borel" in the proof of Theorem 30 and use Theorem 21.

$(2) \Rightarrow(3) B_{p}(X)$ is a topological group, and in particular homogeneous.

$(3) \Rightarrow(4)$ The assumption in (4) implies, in particular, that $f$ is a pointwise limit of $\left\{f_{(1, m)}\right\}_{m \in \mathbb{N}}$. Thus, $f$ is Borel.

\subsection{Bounded Baire-class $\alpha$ images}

Continuous functions and Borel functions are the extremal notions in the Baire hierarchy of functions: A real-valued function $f$ is of Baire class 0 if it is continuous. For $0<$ $\alpha \leq \aleph_{1}, f$ is of Baire class $\alpha$ if $f$ is the pointwise limit of a sequence of functions, each of Baire class smaller than $\alpha$. The function $f$ is Borel if, and only if, $f$ is of Baire class $\aleph_{1}$ (see [23]). A natural question in light of our study is: Which spaces $X$ have the property that each Baire-class $\alpha$ image of $X$ in $\mathbb{N}^{\mathbb{N}}$ is bounded?

Theorem 32. For each $\alpha>0$, the following are equivalent:

(1) Each Baire-class $\alpha$ image of $X$ in $\mathbb{N}^{\mathbb{N}}$ is bounded.

(2) Each Borel image of $X$ in $\mathbb{N}^{\mathbb{N}}$ is bounded.

Proof. Assume that each Baire-class 1 image of $X$ in $\mathbb{N}^{\mathbb{N}}$ is bounded. Baire-class 1 functions are exactly the $F_{\sigma}$-measurable functions.

One way to proceed is to use Lemma 14 , since for each bijectively enumerated family $\mathcal{C}=\left\{C_{n}: n \in \mathbb{N}\right\}$ of closed sets, the corresponding Marczewski function is $F_{\sigma^{-}}$ measurable (and by the proof of Lemma 14, we may assume that for each $x \in X, \mathcal{C}(x)$ is infinite).

However, there is a more direct proof. By Lemma 13, it suffices to prove that $X$ satisfies $\mathrm{U}_{\text {fin }}\left(\mathrm{F}, \mathrm{F}_{\Gamma}\right)$. Assume that $\mathcal{U}_{n}=\left\{C_{m}^{n}: m \in \mathbb{N}\right\}, n \in \mathbb{N}$, are closed covers of $X$ not containing a finite subcover. Define $\Psi: X \rightarrow \mathbb{N}^{\mathbb{N}}$ by

$$
\Psi(x)(n)=\min \left\{m: x \in C_{m}^{n}\right\}
$$

for all $n \in \mathbb{N}$. Each basic open subset of $\mathbb{N}^{\mathbb{N}}$ is an intersection of finitely many sets of the form $O_{m}^{n}=\left\{f \in \mathbb{N}^{\mathbb{N}}: f(n)=m\right\}$. As $\Psi^{-1}\left[O_{m}^{n}\right]=C_{m}^{n} \backslash \bigcup_{k<m} C_{k}^{n}$ is an $F_{\sigma}$ set for all $n$ and $m, \Psi$ is $F_{\sigma}$-measurable. Thus, $\Psi[X]$ is bounded by some $g \in \mathbb{N}^{\mathbb{N}}$. Then $\left\{\bigcup_{m \leq g(n)} C_{m}^{n}: n \in \mathbb{N}\right\}$ is a point-cofinite cover of $X$. 


\section{Closing the circle: Continuous bounded images again}

The proof of Theorem 9 gives us the following analogue of Theorem 2. Say that a set $Y \subseteq$ $\overline{\mathbb{N}}^{\mathbb{N}}$ is bounded if there is $g \in \mathbb{N}^{\mathbb{N}}$ such that for each $f \in Y$ and all but finitely many $n$, $f(n)<\infty$ implies $f(n) \leq g(n)$. This generalizes the standard notions of boundedness in $\mathbb{N}^{\mathbb{N}}$ or $\mathbb{E} \mathbb{F}$.

Theorem 33. The following are equivalent:

(1) Each Borel image of $X$ in $\mathbb{N}^{\mathbb{N}}$ is bounded.

(2) Each continuous image of $X$ in $\overline{\mathbb{N}}^{\mathbb{N}}$ is bounded.

Proof. (1) $\Rightarrow$ (2) Assume that $\Psi: X \rightarrow \overline{\mathbb{N}}^{\mathbb{N}}$ is continuous. Define $d: \overline{\mathbb{N}}^{\mathbb{N}} \rightarrow \mathbb{N}^{\mathbb{N}}$ by $d(x)(n)=x(n)$ if $x(n)<\infty$, and $d(x)(n)=1$ if $x(n)=\infty$. Then $d \circ \Psi: X \rightarrow \mathbb{N}^{\mathbb{N}}$ is Borel, and therefore $d[\Psi[X]]$ is a bounded subset of $\mathbb{N}^{\mathbb{N}}$. Thus, $\Psi[X]$ is a bounded subset of $\overline{\mathbb{N}}^{\mathbb{N}}$.

(2) $\Rightarrow$ (1) Assume that each continuous image of $X$ in $\overline{\mathbb{N}}^{\mathbb{N}}$ is bounded. We first prove that for each bijectively enumerated family $\mathcal{U}=\left\{U_{n}: n \in \mathbb{N}\right\}$ of open sets, $\mathcal{U}$ is a discrete limit of continuous functions. The proof is similar to the proof of Lemma 11. As shown at the end of the proof of Lemma 11, we may assume that no $U_{n}$ is clopen. by

For each $n$, write $U_{n}$ as a union $\bigcup_{m} C_{m}^{n}$ of disjoint clopen sets. Define $\Psi: X \rightarrow \overline{\mathbb{N}}^{\mathbb{N}}$

$$
\Psi(x)(n)= \begin{cases}m, & x \in U_{m}^{n}, \\ \infty, & x \notin U_{n} .\end{cases}
$$

Then $\Psi$ is continuous. Let $g \in \mathbb{N}^{\mathbb{N}}$ bound $\Psi[X]$. For each $n, m$, let

$$
U_{m}^{n}=\bigcup_{i=1}^{\max \{m, g(n)\}} C_{i}^{n} .
$$

For each $m$, define a continuous function $\Psi_{m}: X \rightarrow P(\mathbb{N})$ by

$$
\Psi_{m}(x)=\left\{n: x \in U_{m}^{n}\right\} .
$$

We claim that $\mathcal{U}$ is a discrete limit of the maps $\Psi_{m}, m \in \mathbb{N}$.

Fix $x \in X$. Let $N$ be such that for all $n \geq N, \Psi(x)(n)<\infty$ implies $\Psi(x)(n) \leq g(n)$. For each $n<N$ with $x \in U_{n}$, let $m_{n}$ be such that $x \in U_{m_{n}}^{n}$. Set $M=\max \left\{m_{n}: n<N\right\}$.

Fix $m \geq M$. We show that $n \in \Psi_{m}(x)$ if, and only if, $x \in U_{n}$. One direction follows from $U_{m}^{n} \subseteq U_{n}$. To prove the other direction, assume that $x \in U_{n}$, and consider the two possible cases: If $n<N$, then $x \in U_{m}^{n}$ because $m \geq M \geq m_{n}$, and we are done. Thus, assume that $n \geq N$. Then $\Psi(x)(n) \leq g(n)$, and therefore $x \in \bigcup_{i=1}^{g(n)} C_{i}^{n} \subseteq U_{m}^{n}$.

Thus, for each $x \in X$ there is $M$ such that for all $m \geq M, \Psi_{m}(x)=\mathcal{U}(x)$.

Now, each continuous image of $X$ in $\mathbb{N}^{\mathbb{N}}$ is bounded because $\mathbb{N}^{\mathbb{N}}$ is a subspace of $\overline{\mathbb{N}}^{\mathbb{N}}$. By Hurewicz's Theorem $1, X$ satisfies $\bigcup_{\text {fin }}(\mathrm{O}, \Gamma)$, and by Lemma 12 , so does $\mathcal{U}[X]$. By Lemma 14, each Borel image of $X$ in $\mathbb{N}^{\mathbb{N}}$ is bounded. 
We therefore obtain the aesthetically pleasing result, that the chain of properties

$$
\mathrm{U}_{\text {fin }}\left(\mathrm{B}, \mathrm{B}_{\Gamma}\right) \quad \Longrightarrow \quad \text { hereditarily- } \mathrm{U}_{\mathrm{fin}}(\mathrm{O}, \Gamma) \quad \Longrightarrow \quad \mathrm{U}_{\text {fin }}(\mathrm{O}, \Gamma)
$$

is obtained by requiring bounded continuous images in the chain of subspaces

$$
\overline{\mathbb{N}}^{\mathbb{N}} \supseteq \mathbb{E} \mathbb{F} \supseteq \mathbb{N}^{\mathbb{N}},
$$

respectively.

Acknowledgments. We thank Masami Sakai for his Theorem 6. We also thank Lev Bukovský for useful comments and for making his work [7] available to us prior to its publication, and the referee for his useful comments. A part of the present paper was written when the first author was visiting the Department of Mathematics at the University of Warsaw. We thank Tomasz Weiss for his kind hospitality during that stimulating period.

This research was partially supported by the Koshland Center for Basic Research.

Note. After the present paper was accepted for publication, Bukovský and Šupina [12] devised an alternative, more analytic and less combinatorial, proof of our main Theorem 9.

\section{References}

[1] Arhangel'skiı̌, A.: The frequency spectrum of a topological space and the classification of spaces. Soviet Math. Dokl. 13, 1186-1189 (1972) Zbl 0275.54004 MR 0394575

[2] Arhangel'skiı̌, A.: The frequency spectrum of a topological space and the product operation. Trans. Moscow Math. Soc. 40, 171-206 (1979) Z Zbl 0447.54004 MR 0550259

[3] Arhangel'skiı̌, A., Bokalo, B. M.: The tangency of topologies and tangential properties of topological spaces. Trans. Moscow Math. Soc. 1993, 139-163 Zbl 0798.54002 MR 1256925

[4] Bartoszyński, T., Scheepers, M.: A-sets. Real Anal. Exchange 19, 521-528 (1993/94) Zbl 0822.03028 MR 1282668

[5] Bokalo, B., Malanyuk, O.: Some properties of topological spaces of almost continuous mappings. Mat. Studii 14, 197-201 (2000) Zbl 0979.54021 MR 1814004

[6] Bukovský, L.: Convergence of real functions and covering properties. In: Selection Principles and Covering Properties in Topology, L. Kočinac (ed.), Quaderni Mat. 18, Seconda Università di Napoli, Caserta, 107-132 (2006) Zbl 1158.54309 MR 2395752

[7] Bukovský, L.: On $\mathrm{wQN}_{*}$ and $\mathrm{wQN}^{*}$ spaces. Topology Appl. 156, 24-27 (2008) Zbl 1155.54001 MR 2463820

[8] Bukovský, L., Haleš, J.: On Hurewicz properties. Topology Appl. 132, 71-79 (2003) Zbl 1056.54024 MR 1990080

[9] Bukovský, L., Haleš, J.: QN-spaces, wQN-spaces and covering properties. Topology Appl. 154, 848-858 (2007) Zbl 1117.54003 MR 2294632

[10] Bukovský, L., Recław, I., Repický, M.: Spaces not distinguishing pointwise and quasinormal convergence of real functions. Topology Appl. 41, 25-40 (1991) Zbl 0768.54025 MR 1129696

[11] Bukovský, L., Recław, I., Repický, M.: Spaces not distinguishing convergences of real-valued functions. Topology Appl. 112, 13-40 (2001) Zbl 1067.54028 MR 1815270

[12] Bukovský, L., Šupina, J.: Sequence selection principles for quasi-normal convergence. Topology Appl. 159, 283-289 (2012) 
[13] Chen, H.: Compact-covering maps and $k$-networks. Proc. Amer. Math. Soc. 131, 2623-2632 (2003) Zbl 1025.54022 MR 1974664

[14] Dow, A.: Two classes of Fréchet-Urysohn spaces. Proc. Amer. Math. Soc. 108, 241-247 (1990) Zbl 0675.54029 MR 0975638

[15] Fremlin, D., Miller, A.: On some properties of Hurewicz, Menger and Rothberger. Fund. Math. 129, 17-33 (1988) Zbl 0665.54026 MR 0954892

[16] Filipów, R., Mrożek, N., Recław, I., Szuca, P.: Ideal convergence of bounded sequences. J. Symbolic Logic 72, 501-512 (2007) Zbl 1123.40002 MR 2320288

[17] Gerlits, J., Nagy, Zs.: Some properties of $C(X)$, I. Topology Appl. 14, 151-161 (1982) Zbl 0503.54020 MR 0667661

[18] Gerlits, J., Nagy, Zs.: On Fréchet spaces. Rend. Circ. Mat. Palermo Suppl. 18, 51-71 (1988) Zbl 0678.54017 MR 0958724

[19] Hurewicz, W.: Über Folgen stetiger Funktionen. Fund. Math. 9, 193-204 (1927) JFM 53.0562.03

[20] Jasinski, J., Recław, I.: Ideal convergence of continuous functions. Topology Appl. 153, 35113518 (2006) Zbl 1105.54008 MR 2270601

[21] Jasinski, J., Recław, I.: Spaces with the ideal convergence property. Colloq. Math. 111, 43-50 (2008) Zbl 1143.54008 MR 2353929

[22] Just, W., Miller, A., Scheepers, M., Szeptycki, P.: The combinatorics of open covers II. Topology Appl. 73, 241-266 (1996) Zbl 0870.03021 MR 1419798

[23] Kechris, A.: Classical Descriptive Set Theory. Grad. Texts in Math. 156, Springer (1994) Zbl 0819.04002 MR 1321597

[24] Kočinac, Lj.: Selected results on selection principles. In: Proceedings of the 3rd Seminar on Geometry and Topology, Sh. Rezapour (ed.), Tabriz, 71-104 (2004) MR 2090207

[25] Kočinac, Lj.: Selection principles related to $\alpha_{i}$-properties. Taiwanese J. Math. 12, 561-572 (2008) Zbl 1153.54009 MR 2417134

[26] Menger, K.: Einige Überdeckungssätze der Punktmengenlehre. Sitzungsber. Wiener Akad. 133, 421-444 (1924) JFM 50.0129.01

[27] Miller, A.: A hodgepodge of sets of reals. Note Mat. 27, 25-39 (2007) Zbl 1195.03049 MR 2406153

[28] Nowik, A.: Additive properties and uniformly completely Ramsey sets. Colloq. Math. 82, 191-199 (1999) Zbl 0942.03050 MR 1735477

[29] Recław, I.: Every Luzin set is undetermined in the point-open game. Fund. Math. 144, 43-54 (1994) Zbl 0809.04002 MR 1271477

[30] Recław, I.: Metric spaces not distinguishing pointwise and quasinormal convergence of real functions. Bull. Polish Acad. Sci. Math. 45, 287-289 (1997) Zbl 0897.54013 MR 1477547

[31] Rubin, M.: A Boolean algebra with few subalgebras, interval Boolean algebras and retractiveness. Trans. Amer. Math. Soc. 278, 65-89 (1983) Zbl 0524.06020 MR 0697061

[32] Sakai, M.: A special subset of the real line and regularity of weak topologies. Topology Proc. 23, 281-287 (1998) Zbl 0977.54003 MR 1743815

[33] Sakai, M.: The sequence selection properties of $C_{p}(X)$. Topology Appl. 154, 552-560 (2007) Zbl 1109.54014 MR 2280899

[34] Scheepers, M.: A sequential property of $C_{p}(X)$ and a covering property of Hurewicz. Proc. Amer. Math. Soc. 125, 2789-2795 (1997) Zbl 0881.54038 MR 1396994

[35] Scheepers, M.: Combinatorics of open covers I: Ramsey theory. Topology Appl. 69, 31-62 (1996) Zbl 0848.54018 MR 1378387

[36] Scheepers, M.: $C_{p}(X)$ and Arhangel'skiı̌'s $\alpha_{i}$ spaces. Topology Appl. 89, 265-275 (1998) Zbl 0930.54017 MR 1645184 
[37] Scheepers, M.: Sequential convergence in $C_{p}(X)$ and a covering property. East-West J. Math. 1, 207-214 (1999) Zbl 0976.54016 MR 1727383

[38] Scheepers, M.: Selection principles and covering properties in topology. Note Mat. 22, 3-41 (2003) Zbl 1195.37029 MR 2112729

[39] Scheepers, M., Tsaban, B.: The combinatorics of Borel covers. Topology Appl. 121, 357-382 (2002) Zbl 1025.03042 MR 1908999

[40] Shakhmatov, D.: Convergence in the presence of algebraic structure. In: Recent Progress in General Topology, II, North-Holland, Amsterdam, 463-484 (2002) Zbl 1029.54003 MR 1970008

[41] Tsaban, B.: Some new directions in infinite-combinatorial topology. In: Set Theory, J. Bagaria and S. Todorčević (eds.), Trends in Mathematics, Birkhäuser, 225-255 (2006) Zbl 1113.54002 MR 2267150

[42] Vinokurov, V.: Strong regularizability of discontinuous functions. Soviet Math. Dokl. 281, 265-269 (1985) MR 0785271

[43] Zdomskyy, L.: A semifilter approach to selection principles. Comment. Math. Univ. Carolin. 46, 525-539 (2005) 